\title{
磁性材料在同步辐射及自由电子激光中的应用
}

\author{
何永周，周巧根 \\ (中国科学院上海应用物理研究所, 上海 201204)
}

\begin{abstract}
摘 要: 同步辐射与自由电子激光都是相对论高能电子束产生的电磁辐射光, 在国民经济、科学技术研究和国防军 事等领域中有着广泛应用。各种永磁插入件是同步辐射光源及自由电子激光装置的关键设备之一, 磁性材料特别是 永磁体磁特性及质量对插入件的磁场品质、磁场峰值、磁场稳定性和运行方案等都有着重要影响。本文综述了同 步辐射及自由电子激光特征，介绍了磁性材料在同步辐射及自由电子激光插入件中的应用情况。
\end{abstract}

关 键 词: 同步辐射; 自由电子激光; 永磁插入件; 磁性材料

中图分类号: O57; O441; TM271 文献标识码: A

\section{Application of Magnetic Materials in Synchrotron Radiation and Free Electron Laser}

\author{
HE Yong-Zhou, ZHOU Qiao-Gen
}

(Shanghai Institute of Applied Physics, Chinese Academic of Sciences, Shanghai 201204, China)

\begin{abstract}
The synchrotron radiation and free electron laser are electromagnetic radiation light sources which are both generated by the relativistic electron beam. Both sources have been widely used in the national economic, scientific and technical researches, national defense and military fields. The insert device made of permanent magnet is one of the key equipments of the synchrotron radiation light source and the free electron laser facility. Magnetic properties and quality of magnetic materials especially have significant influence on magnetic field quality, magnetic field peak value, magnetic field stability and operation scheme of insert devices. This paper reviewed characteristics of synchrotron radiation and free electron laser, and their conceptual application.
\end{abstract}

Key words: synchrotron radiation; free electron laser; permanent magnet insert device; magnetic materials.

光源发光是由于光源中粒子如电子、原子、分 子等运动产生, 主要有三种方式: 热运动、跃迁辐射 和带电粒子加速运动。热运动产生光是生活中最常 见的, 太阳光、蜡烛就是很好例子, 此类光随着温度 变化会改变颜色。跃迁辐射光是由物质粒子如分子、 原子等通过能级间跃迁产生, 粒子自发从高能级激 发态 $E_{2}$ 向低能级基态 $E_{1}$ 跃迁, 同时辐射出频率为 $v=\left(E_{2}-E_{1}\right) / h$ 的光子, 这种过程称为自发辐射。当 外来光子入射时, 会引发原子以一定概率迅速从能 级 $E_{2}$ 跃迁到能级 $E_{1}$, 同时辐射一个与外来光子频
率、相位、偏振态以及传播方向都相同的光子，这 个过程称为受激辐射, 这种在受激辐射过程中产生 并被放大的相干光就是激光。带电粒子加速运动产 生光通常称为同步辐射光和自由电子激光, 称为基 于加速器光源, 原子核反应堆切伦科夫辐射也属于 这种。

插入件是同步辐射和自由电子激光装置的关键 设备之一 ${ }^{[1-3]}$ 。1985 年, 美国伯克利国家实验室 Halbach 首先利用稀土永磁研制出 Halbach 波荡器插 入件 ${ }^{[4]}$, 大大缩短了插入件的周期长度。自上世纪 
80 至 90 年代, 国内外研究机构相继研制成功多种 永磁插入件, 有力推动了同步辐射、自由电子激光 等高能物理的发展。多种性能各异永磁插入件在线 运行为多项科学实验提供了有力保障。随着高技术 发展, 各类永磁 Halbach 磁路装备 ${ }^{[5-7]}$ 也在众多领域 如雷达/通讯、航空/航天、新武器、能源、交通、 医疗等行业获得越来越广泛应用。

磁性材料 ${ }^{[8]}$, 特别是永磁材料 ${ }^{[9]}$ 是插入件磁场源, 对插入件整机综合性能有着决定性的影响。本文综 述了基于加速器的同步辐射及自由电子激光相对论 高能电子束产生电磁辐射光的原理及其在现代社会 中的应用, 同时介绍了各种磁性材料在同步辐射及 自由电子激光插入件中的应用情况。

\section{1 高能加速器光源装置}

\section{1 同步辐射}

同步辐射是速度接近光速带电粒子在磁场中沿 弧形轨道运动时放出的自发及准相干电磁辐射光, 最初在同步加速器上观察到, 又被称为同步辐射 (图 1) ${ }^{[10]}$ 。长期以来, 同步辐射阻碍了加速器粒子能 量提高而不受欢迎，但人们很快发现同步辐射是从 远红外到 X 波段范围连续脉冲光源, 具有高强度、 高准直、高度极化和特性可控等优异性能, 可用它 开展其它光源无法实现的许多前沿科学技术研究。

同步辐射光源是产生同步辐射光以满足研究与 应用的大科学装置, 包括注入器、储存环和光束线 站三部分: 注入器产生电子, 并将电子加速到所需 额定能量, 再引入储存环; 储存环保持电子以额定 能量在其中稳定回转运动并发出同步辐射光; 光束 线站将光引入相关实验站进行实验和应用 ${ }^{[11]}$ 。自上 世纪四十年代诞生以来, 同步辐射装置的设计建造 经历了三代: 第一代是寄生于对撞机的兼用机; 第 二代是基于弯铁并带少量插入件同步辐射储存环专 用机; 第三代是主要基于各种高性能插入件同步辐

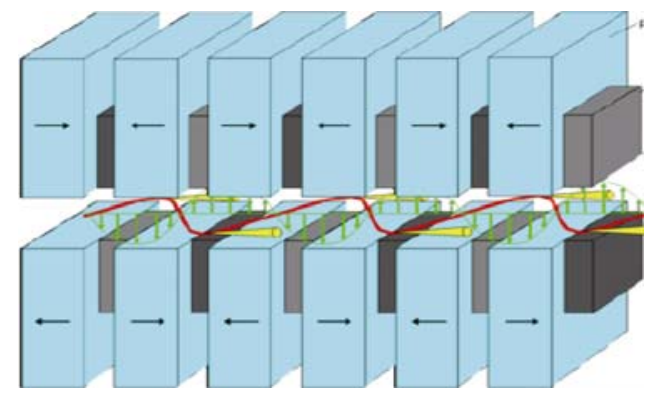

图 1 同步辐射光 ${ }^{[4]}$

Fig. 1 Synchrotron radiation light ${ }^{[4]}$
射储存环专用机。目前世界上已建成和即将建成的 三代光源总计约 65 台。三代光源间最主要区别是电 子束发射度, 还有一个显著差别是插入件数量, 插 入件较弯铁同步辐射光具有更高亮度而获得极为广 泛应用，插入件性能是直观表征同步辐射光源性能 优劣重要指标之一。第三代光源大量使用插入件, 尤其是波荡器, 使得光亮度较二代至少提高了两个 量级, 比 $\mathrm{X}$ 光要亮一亿倍以上。图 2 为我国上海同 步辐射光源(SSRF)布局图。

同步辐射是生命、材料、环境、物理、化学、医 药、地质等学科基础应用研究中一种最先进的不可替 代工具, 并且在电子、医药、石油、化学、生物和微 细加工等工业领域方面也具有重要应用价值。

\section{2 自由电子激光}

自由电子激光是激光家族新成员，工作介质是 “自由的电子”, 称为自由电子激光 ${ }^{[12]}$, 特点是波长 和脉冲结构可灵活设计。其原理是: 当电子束与外 来种子激光同时通过波荡器时，电子束在波荡器磁 场作用下发生横向周期运动，这一运动又与外来激 光的磁分量相互作用产生轴向力 (称作质动力势), 质动力势也是周期性的，电子在它驱动下便形成纵 向群聚的团束，电子束团的光辐射与种子激光相干 叠加, 当满足共振关系及其他条件时, 激光光场从 电子束中获取能量实现受激光放大。利用这一思想 设计的激光装置称为自由电子激光器(FEL)。

自由电子激光装置包括电子枪、加速器、波荡 器、实验站等, 其中电子枪产生电子, 加速器使电子 加速运动, 波荡器产生相干光, 实验站供用户应用。 自由电子激光装置根据种子激光来源及波荡器分为 四种 ${ }^{[13]}$ : 放大型(种子激光和电子束一起进入波荡 器)、自放大(波荡器自发辐射作为种子激光)、振荡 型(波荡器自发反射光作为种子激光)、相干谐波型 (光学速调管替代波荡器)。四种自由电子激光各有 优缺点，自放大型结构简单、波长连续可调、易实

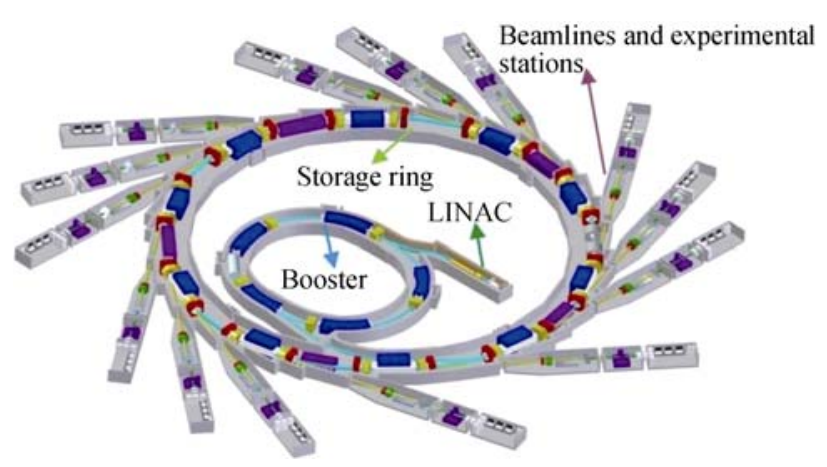

图 2 中国大陆 SSRF 布局 ${ }^{[11]}$

Fig. 2 Layout of SSRF for China ${ }^{[11]}$ 
现等特点逐渐成为主要发展方向之一。

自由电子激光发展方向为: (1)缩短波长, 由于 技术困难, 目前自由电子激光装置主要工作在远红 外与红外区，随着技术发展, FEL 将向短波方向推 动; (2)提高功率, 这主要是出于军事目的(如定向能 武器和军事通信); (3)小型化, 小型化可以使 FEL 获 得广泛工业应用; (4)提高转换效率, 目前 FEL 能量 转换效率还很低 $(10 \%$ 20\%)。目前世界上已建成 FEL 近 100 台, 中科院上海应用物理研究所、工程 物理研究院、中国科技大学、中科院北京高能物理 研究所等单位建有不同类型 FEL 装置(图 3)。

从 1971 年美国 Mady 首先提出 FEL 概念以来, FEL 以无可比拟的性能优点, 在信息、超导、凝聚 态、物理和化学等多个领域具有极为重要应用价值。 如自由电子激光高功率、宽光谱等特点, 可用于物 质提纯、受控核聚变、同位素分离和等离子体加热 等。自由电子激光高效率、短脉冲等优点, 在工业 上也有广阔应用前景, 如半导体、集成电路、激光 加工、基础研究、医疗、空间科学等。在军事上, 自 由电子激光器可以成为强激光武器, 是反洲际导弹 主要潜在手段之一。在毫米波段, 自由电子激光器 是唯一有效强相干信号源, 在激光雷达、反隐形目 标等研究中具有不可替代的应用价值。

\section{3 插入件}

弯铁、扭摆器和波荡器产生三种同步辐射光 ${ }^{[14-16]}$, 其机制见图 4, 扭摆器和波荡器统称插入件。

图 5 为波荡器辐射波谱, 从图中可以看到, 波荡

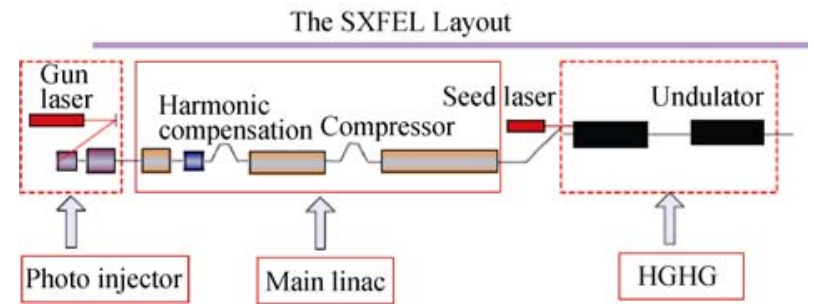

图 3 我国 SXFEL 自由电子激光装置布局 ${ }^{[17]}$

Fig. 3 Layout of SXFEL free electron laser facility for China ${ }^{[17]}$
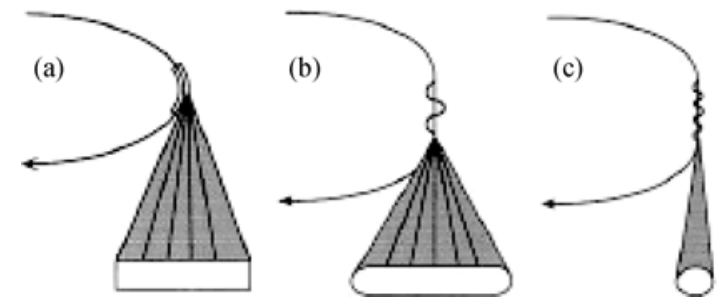

图 4 三种同步辐射光 ${ }^{[15]}$

Fig. 4 Three kinds of synchrotron radiation light ${ }^{[15]}$ (a) Bending iron; (b) Wiggler; (c) Undulator
器辐射光亮度远大于弯铁和扭摆器, 扭摆器和弯铁辐 射可覆盖更加宽阔波谱范围, 波荡器辐射代表了同步 辐射最高性能,波荡器约占插入件总数的 $95 \%$ 以上。

插入件Halbach磁结构特点是具有磁场单边增强 效应，较传统永磁阵列更接近于正弦磁场分布,理想 Halbach磁结构见图6, 标准结构磁力线分布见图7。

插入件又分为电磁型和永磁型，而永磁插入件 因众多优点占绝大多数。永磁插入件又分为纯永磁 型PPM和混合型 $\mathrm{Hyb}$ ，在这二个磁结构基础上可衍 生成APPLE、HELIOS、Delta等多种磁结构，不同磁

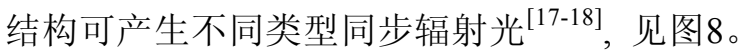

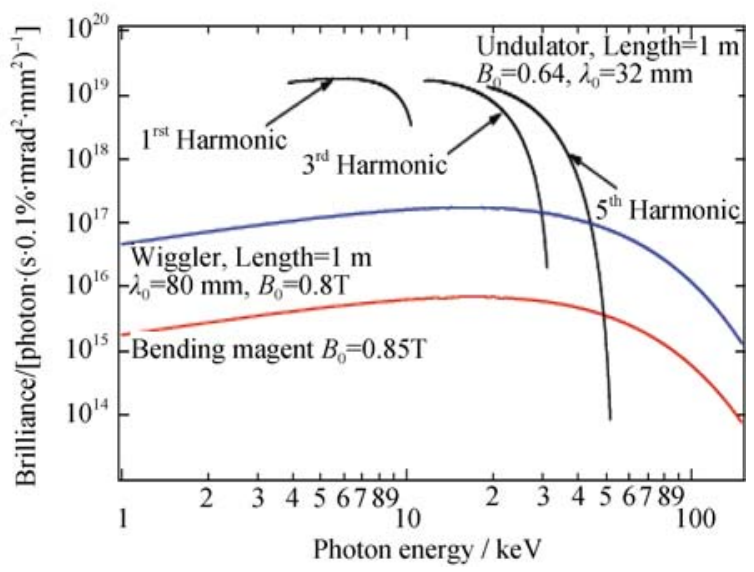

图 5 三种同步辐射光谱 ${ }^{[15]}$

Fig. 5 Spectra of synchrotron radiation ${ }^{[15]}$

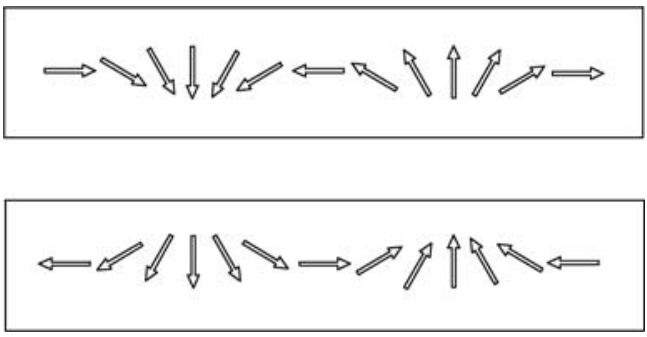

图 6 理想 Halbach 磁路 ${ }^{[1]}$

Fig. 6 Ideal Halbach magnetic circuit ${ }^{[1]}$

PPM

Hybrid
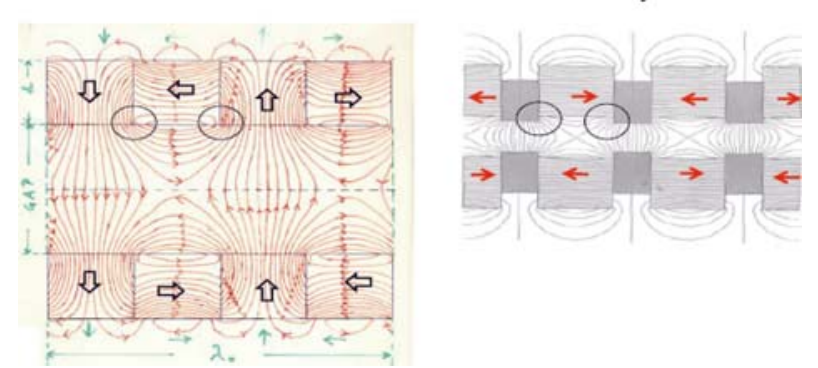

图 7 标准 Halbach 磁结构磁力线分布 ${ }^{[1]}$

Fig. 7 Flux distribution of standard Halbach structure ${ }^{[1]}$ 


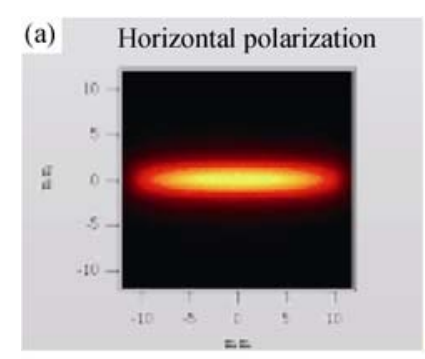

(c) Linear inclined polarization
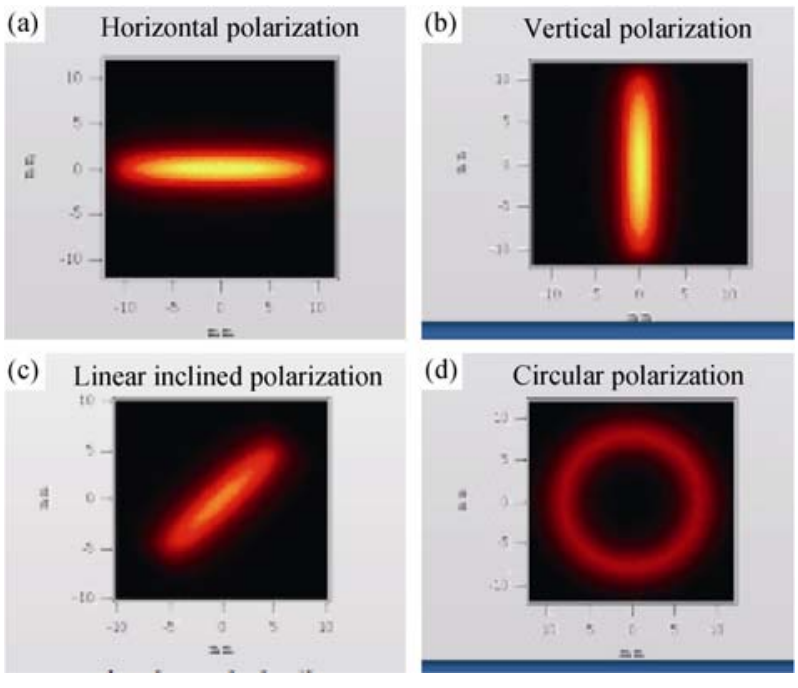

图 8 同步辐射光模式 ${ }^{[17]}$

Fig. 8 Synchrotron radiation light mode $\mathrm{e}^{[17]}$

(a) Horizontal; (b) Vertical; (c) Line inclined; (d) Elliptical polarization

永磁波荡器由上下两排 “Halbach” 永磁阵列构 成, 在中平面上产生接近正弦垂直磁场, 电子通过 时在水平面上作扭摆运动或波荡运动, 产生水平极 化同步辐射光, 上下磁间隙一般是可调节以得到不 同峰值磁场。目前常用永磁插入件有(图9): 平面波 荡器、真空插入件 (又分真空波荡器IVU, 真空扭摆 器IVW $)^{[14]}$ 、椭圆极化波荡器EPU ${ }^{[16]}$ 、低温永磁波荡 器CPMU ${ }^{[19]}$ 。

\section{2 插入件用磁性材料}

磁性材料种类繁多且应用十分广泛, 主要分为 二类: 一类是磁滞回线为特征的磁性材料, 如永磁、 软磁、矩磁等; 另一类是以交叉偶合为特征的磁性 材料, 如磁致伸缩材料、磁光材料、磁热材料等。 用途最大是第一种, 特别是永磁和软磁。

商业永磁材料有铝镍钴、铁氧体、钐钴、钕(镨) 铁喼等 ${ }^{[9]}$, 应用于插入件的永磁体有钐钴和稀土钕 (镨)铁喼 ${ }^{[20-22]}$ 。钐钴又分为 $1: 5$ 型、 $2: 17$ 型, $\mathrm{SmCo}_{5}$
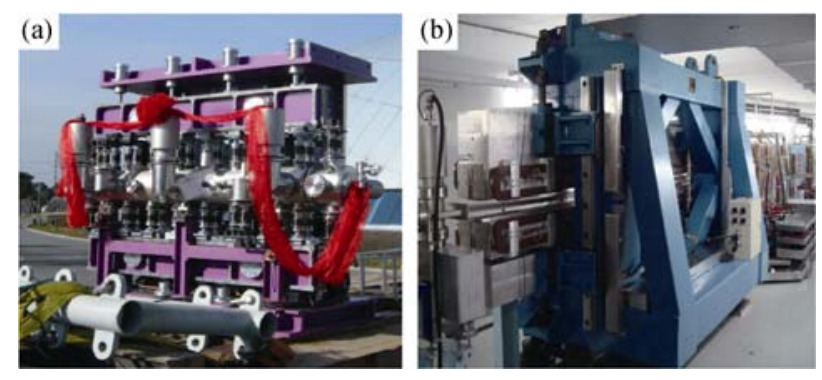

图 9 典型插入件 ${ }^{[17]}$

Fig. 9 Typical insert device ${ }^{[17]}$

(a) In-vacuum undulator; (b) Elliptically polarized undulator
剩磁为 $0.9 \sim 1.0 \mathrm{~T}, \mathrm{Sm}_{2} \mathrm{Co}_{17}$ 剩磁为 $1.0 \sim 1.14 \mathrm{~T}$, 钐钴具 有耐电磁辐射、稳定性好、温度系数小等优点, 目 前日本信越化工R32SH、德国真空冶炼225TP、中 国杭州永磁集团 $30 \mathrm{H}$ 等高性能钐钴永磁体广泛应用 于国内外真空波荡器及加速器永磁组件中。非真空 常规波荡器, 如平面型波荡器、EPU和CPMU主要使 用钕(镨)铁硼, 常温剩磁1.18 1.35T, 典型的是超高 内禀矫顽力 $\mathrm{SH}$ 系和极高内京矫顽力 $\mathrm{EH}$ 系等, 这些 永磁体常温在保持较高剩磁基础上, 内禀矫顽力也 相当可观, 在较大磁间隙可耐电磁辐射。相对钐钴, 钕(镨)铁嗍具有剩磁高、成本低等优点, 但抗电磁辐 射、耐腐蚀性和长期稳定性等欠佳, 主要应用于常 温真空外波荡器中。CPMU为获得优异低温磁场, 在保持常温磁场稳定性基础上, 多使用高剩磁高内 禀矫顽力的 $\mathrm{H}$ 系列或接近 $\mathrm{SH}$ 的钕(镨)铁硼; 纯镨铁 嗍低温磁性能有较大提升空间，但设计与制造技术

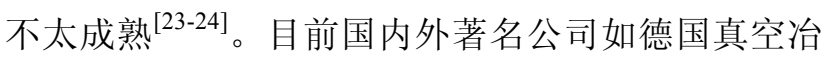
炼、日本日立、中国杭州永磁集团等研制的钕铁嗍 已广泛应用于国内外永磁插入件。

商业软磁材料有铁氧体、非晶(如铁基与钴基 等)、纳米晶(如铁基纳米晶 $\mathrm{FeCuB}$ )和晶态(如钴铁与 粉末合金等 $)^{[8]}$ 。软磁大多数呈各向同性, 部分呈现 较小的各向异性特征。全过程技术 $M-H$ 特征呈非 线性, 较低磁场 $H$ 下 $M-H$ 有一定线性特征。软铁 性能各异, 用途差别也较大, 用于静态波荡器主要 有纯铁(DT4C)、低碳钢、钴钒铁等, 三种软磁在低 $H$ 下磁化曲线近线性, 最大饱和磁场分别为 $2.15 \mathrm{~T}$ 、 $2.08 \mathrm{~T} 、 2.35 \mathrm{~T}$ 。纯铁和低碳钢主要用于波荡器中, 扭 摆器或低温永磁波荡器为达到较高磁场峰值, 一般 采用高饱和磁场及耐高温的钴钒铁。

\section{1 插入件磁铁设计}

同步辐射及自由电子激光插入件磁铁设计目标 是围绕高能电子加速器特征及用户科学实验要求确 定插入件整体性能参数, 主要目标有: (1)确定工作 磁间隙 $g$, 周期长 $\lambda_{0}$, 磁场峰值 $B_{0}$, 插入件参数 $K$, 永磁体与软磁铁几何参数; (2)优化端部永磁体及软 磁铁结构设计, 使一次与二次积分不随磁间隙变化 有较大变化; (3)考虑插入件工作环境, 确定永磁体 安全工作点; (4)插入件磁场技术指标及相应永磁体 与软磁磁性能参数及品质要求。插入件磁铁设计中, 磁性材料特别是永磁材料的技术选型、磁场品质、 外形尺寸以及镀层外观等对插入件整机磁场峰值、 磁场均匀性和磁场稳定性等有着极为重要影响。

插入件磁铁设计计算仿真主要采用有限元法和 半解析数值方法。商业有限电磁场软件有Ansys、 
Flux3D、Opera3D等, 其中Opera对电磁矢量场计算 更专业，方便建立较复杂模型，不需编制程序语言， 该软件在电磁计算领域获得了广泛应用。Radia是欧 洲同步辐射装置(ESRF)开发的专门用于同步辐射电 磁铁与插入件磁场设计计算的程序, 计算采用边界 积分方程法, 属于半解析数值方法, 它是寄生在 Mathematica 软件内代码, 实际使用时可以结合并 发挥Opera和Radia二种计算方法的优点。

\section{2 插入件永磁体}

\subsection{1 磁性能基本参数}

插入件永磁体设计需要的磁性能参数主要有: $B_{\mathrm{r}} 、 H_{\mathrm{ci}} 、 H_{\mathrm{cb}} 、 H_{\mathrm{k}} 、 \mu_{\mathrm{r}}$ 。 $B_{\mathrm{r}} 、 H_{\mathrm{cb}} 、 \mu_{\mathrm{r}}$ 主要影 响波荡器磁场峰值, $H_{\mathrm{ci}}$ 与 $H_{\mathrm{k}}$ 主要影响波荡器能否 安全工作, 磁场数值计算主要涉及上述几个参数, 同时计算时还要参考剩磁温度系数 $\alpha$ 和内亭矫顽力 温度系数 $\beta$ 。插入件永磁体开路条件下, 磁场强度 $H$ 、磁感应强度 $B$ 、剩余磁化强度 $M$ 三者间关系为 $B=\mu_{0}(H+M)$, 插入件永磁体是各向异性的, 平 行取向时 $B=\mu_{0}(\chi H+H)+B_{\mathrm{r}}$, 垂直取向时 $B_{\perp}=$ $\mu_{0}\left(\chi_{\perp} H_{\perp}+H_{\perp}\right)+B_{r, \perp}$, 较大垂直取向磁场存在是不 允许的(垂直取向比较小, 在精度不高永磁装置和设 备中可以忽略), 典型永磁体部分磁性能如图10。

\subsection{2 外磁场品质}

永磁体磁场品质指标有: 磁矩 $M_{\mathrm{r}}$ 一致性、磁 化偏角 $T 、 \mathrm{~N} / \mathrm{S}$ 极磁场对称性 $C_{\mathrm{NS}}$ 、微观磁场积分 等 ${ }^{[25-27]}$ 。

磁矩一致性表示插入件几百甚至近千件永磁体 工作点对应 $M_{\mathrm{r}}$ 值应控制在一定范围。

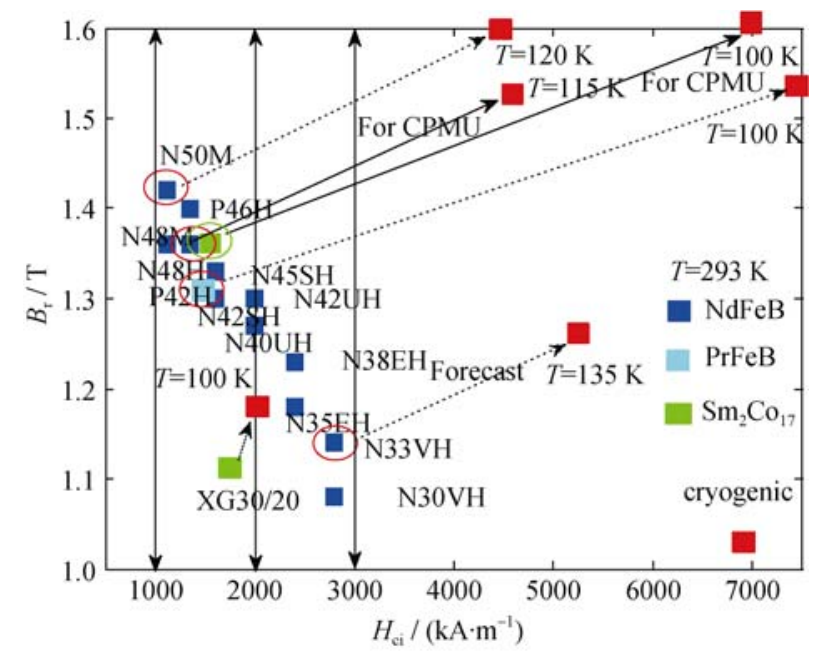

图 10 国产永磁体磁性能 ${ }^{[19]}$

Fig. 10 Magnetic properties of the permanent magnet for China $^{[19]}$
磁化偏角计算公式为,

$$
T=\arctan \sqrt{M_{x}^{2}+M_{y}^{2}} / M_{z}
$$

插入件永磁体一般为块状,少数为铸形, 三维磁 矩有 $M_{x} 、 M_{y} 、 M_{z}, M_{z}$ 为主磁矩。

$\mathrm{N} / \mathrm{S}$ 极磁场对称性计算公式为,

$$
C_{\mathrm{NS}}=\left\{\left[2\left(\left|B_{\mathrm{N}}\right|-\left|B_{\mathrm{S}}\right|\right)\right] /\left[\left(\left|B_{\mathrm{N}}\right|+\left|B_{\mathrm{S}}\right|\right)\right]\right\} \times 100 \%
$$

微观磁场积分指永磁体周围空间三维磁场 $B_{x}$ 、

$B_{y} 、 B_{z}$ 积分的分布形态。

理想情况波荡器磁场波形呈正弦, 如果磁矩一 致性不好，正弦波正值和负值将不能完全抵消，从 而对波荡器磁性能产生不利影响(如磁场相位误差), 多数情况下 $M_{\mathrm{r}}$ 一致性要求控制在 $\pm 1.0 \%$ 以内。如果 永磁体 $T$ 为零, 波荡器磁路二侧单边将无磁荷累积 分布, 这有利于波荡器整机磁场品质提高, 但一般 情况永磁体或多或少有一定 $T$, 从而在磁路二侧单 边产生不均匀的正负磁荷。因此实际工程条件下, 绝大部分永磁体 $T$ 控制在 $1^{\circ}$ 以内 $($ 也有少数特殊设 计的永磁体反而要求有一定的大磁化偏角), 且需 对每件永磁体进行较为精确的三维磁矩测量, 通过 排序使得Halbach磁路一边的磁荷正负大体抵消。永 磁体 $\mathrm{N} / \mathrm{S}$ 极磁场 $C_{\mathrm{NS}}$ 如对称性不好, 将对波荡器局 部磁性能一致性及均匀性产生不利影响。微观磁场积 分对工作在小磁间隙波荡器整机磁场有重要影响。

\subsection{3 机械尺寸及镀层}

插入件永磁体绝大多数设计成块状, 行业俗称 “磁化块”。插入件的高精度安装对几百件永磁体元 件机械尺寸提出了严格要求，见图11。平面型波荡 器如真空外波荡器, 真空波荡器, 低温永磁波荡器 等, 标准磁化块取向方向尺寸精度控制较为严格, 一般镀层成品控制在 $0.05 \mathrm{~mm}$ 内, 永磁体取向方向 厚度越小，控制精度越高。椭圆极化波荡器磁化块所 有方向尺寸公差及相邻面垂直度控制均要求比较高,

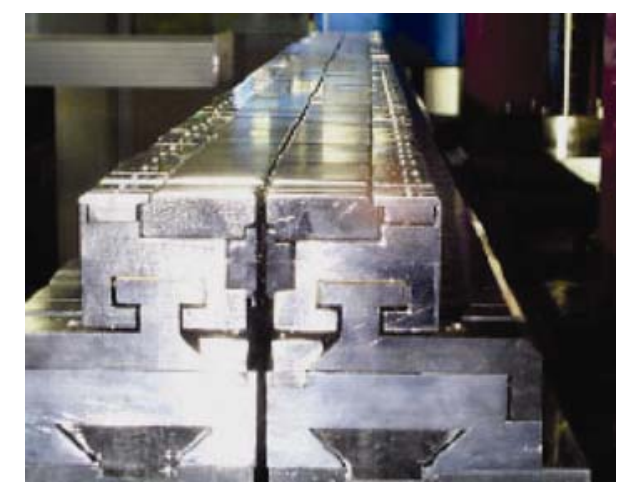

图 11 波荡器内大梁永磁体阵列 ${ }^{[17]}$

Fig. 11 Permanent magnets array of undulator beam ${ }^{[17]}$ 
一般控制在 $0.05 \mathrm{~mm}$ 内, 垂直度控制在 $0.04 \mathrm{~mm}$ 内, 磁化块越小, 机械尺寸及垂直度控制精度要求越高。

插入件永磁体对镀层共性要求是结合力、外观 优良, 为保证永磁体良好机械尺寸及形位公差, 也 要求磁化块镀层无明显 “边角效应”。真空插入件也 要求镀层密封后永磁体常温出气率 ${ }^{[28]}$ 达到 $1.33 \times$ $10^{-8} \mathrm{~Pa}$ 数量级, 这对永磁体镀层提出了极高要求。 真空波荡器及低温永磁波荡器等永磁体工作在较小 的磁间隙下, 永磁体将承受高能电子束产生的各种 复杂的直接与间接的电磁辐射, 普通 $\mathrm{NiCuNi}$ 一般不 能满足要求, 为此需要对永磁体实施特殊镀层处理, 如 TiN密封等。图12为典型插入件永磁体镀层。

\section{3 插入件用软磁}

软磁材料元件在混合型插入件主要对磁场源 (永磁体或通电线圈)起着聚磁及放大作用。插入件 设计时主要关心软磁的磁化曲线 $M-H$ 、相对磁导 率 $\mu_{\mathrm{r}}$ 、饱和磁感应场强度 $M_{\mathrm{s}}$ 。此外软磁铁外形尺 寸精度、表面光洁性、表面毛刺和加工残余应力等 对其聚磁能力及效果有重要影响。软磁材料元件加 工过程中不得有含硫冷却液, 表面去毛刺并清洗, 镀层后进行真空退火以去除加工残余应力。

\section{4 未来展望}

磁性材料特别是永磁材料磁性能提升及其综合 特性研究成果有力促进了插入件技术不断发展, 同时 同步辐射及自由电子激光高能物理的发展也对磁性 材料提出了越来越高的要求。未来插入件伴随永磁 体及软铁技术进步有望在以下方面继续获得进展。

\subsection{1 永磁体磁性能}

目前商业永磁体磁性能还没有达到理论值, 随 着钐钴及钕铁硼常温磁性能的提升, 未来插入件磁 性能也将不断获得进步。如钐钴理论剩磁1.25T, 目 前国产钐钴磁性能: $B_{\mathrm{r}}=1.10 \mathrm{~T} @ H_{\mathrm{cj}}=1600 \mathrm{kA} / \mathrm{m}$, 剩 磁还有不少提高空间。钕铁嗍理论剩磁 $1.60 \mathrm{~T}$, 目前 国内外: $B_{\mathrm{r}}=1.35 \mathrm{~T} @ H_{\mathrm{cj}}=1600 \mathrm{kA} / \mathrm{m}$ 。

镨铁嗍永磁体在强磁场低温永磁波荡器中有重
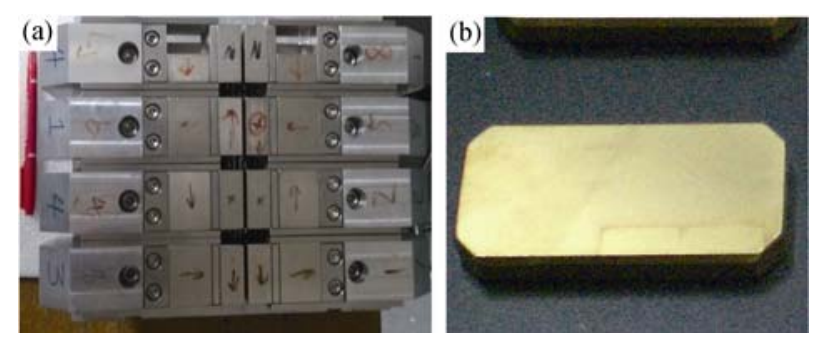

图 12 典型永磁体镀层

Fig. 12 Typical coating of permanent magnet (a) $\mathrm{NiCuNi}$; (b) TiN
要应用前景, 目前国内外制备的镨铁硼磁性能、磁 场均匀性、镀层密封还有不少问题，随着镨铁硼制 备技术的不断成熟, 低温永磁波荡器也将获得较大 发展空间。

\subsection{2 新技术永磁体}

近十年来, 稀土永磁体设计与制造技术获得了 长足进展, 出现了一些新概念技术制备的永磁体, 如晶界扩散钕铁嗍、微晶钕铁硼等, 这些永磁体磁 性能较常规永磁体磁性能有大幅度提高。通过与永 磁体公司密切合作，有望研制出基于更高性能永磁 体, 对新型插入件的设计研制有重要推动作用。国 产钐钴目前还采用落后的机械或气流磨制备合金粉 末, 如果掌握先进的氧化还原粉末制备工艺, 钐钴 磁性能有望获得重大突破性进展。

\subsection{3 永磁体均匀性}

磁性材料特别是永磁材料磁场均匀性对插入件 整机磁场品质有重要影响, 未来随我国磁性材料制 备水平的提升, 基于高均匀磁性材料元件、插入件 对同步辐射及自由电子激光发展将有着重要推动作 用。如目前国产钐钴采用传统粉末冶金工艺制备, 存在粉末颗粒形状多样且尺寸分布不具正态分布特 征, 造成磁化偏角和磁场积分等不够理想, 对插入 件整机磁场性能有不利影响。

\subsection{4 永磁体稳定性}

工作在小磁间隙下的真空插入件, 因相对论电 子束能量的强大电磁辐射及电子直接冲击, 对永磁 体长期稳定性提出了苛刻要求。影响插入件永磁体 长期稳定性主要因素有: 微结构均匀性、内禀矫顽 力及方形度、温度系数、工作点设计合理性、镀层 材料及质量、电子束能量及密度等。伴随着我国永 磁体制造及应用水平的提高, 我国未来插入件永磁 体稳定性也将有大幅度改善。

\subsection{5 永磁体镀层}

随着我国同步辐射及自由电子激光技术的不断 发展, 对永磁插入件真空性能提出了越来越高的要 求, 目前我国制备的永磁体镀层真空密封技术离国 外先进水平还有不少差距，制约了我国小磁间隙永 磁插入件的研制与在线稳定运行，在小磁间隙下永 磁体将承受复杂的外加环境, 如高能电子束冲击、 强电磁辐射、高退磁场、热负载等, 这对永磁体包 括磁性能在内的镀层密封技术提出了很高的要求。

\subsection{6 新型软磁材料}

目前常规电工纯铁等软磁在较高磁场下容易饱 和, 新型软磁材料设计研制显得十分重要, 如具有 极高低温饱和磁化强度稀土单晶体的制备, 常温综 合性能优异和高饱和磁化强度的纳米晶软磁等。新 
型软磁材料元件的设计与研制将推动高性能插入件 技术进一步发展。

\section{3 总结}

研究、设计和研制永磁体主要国家有中国、日 本、德国，其他国家比例很小。我国是永磁体生产 大国，国民经济及国防等领域需要各种永磁体，我 国在基础研究、设计工艺、实验制备、生产技术等 环节都具有相当实力。但除部分种类永磁体外(如铁 氧体), 我国生产的稀土钐钴和钕(镨)铁硼在高端领 域应用研究经验与技术储备较少, 很多领域处于空 白状态。日、德二国钐钴系和钕(镨)铁嗍永磁体元 件产量虽仅占世界 $20 \%$ 左右，但掌握了应用领域众 多关键制造技术，近 $90 \%$ 永磁体应用于高、精、尖 领域。

磁性材料元件特别是永磁材料是各种插入件的 “心脏发动机”。在这方面国产永磁体差距是多方面 的, 主要体现在: 磁性能、磁矩一致性、微观均匀性、 稳定性、磁场测量、镀层密封和外观质量等。目前 欧洲、美国、日本等发达国家同步辐射光源及自由 电子激光装置插入件安装的大量高品质永磁体主要 来自日本信越、日立金属、德国真空冶炼等国际著 名永磁公司，国外同步辐射光源及自由电子激光装 置研究机构长期和这些永磁公司保持合作关系, 对 插入件用永磁体进行了系统、深入、细致的研究, 获 得了大量有价值的学术及应用成果, 促进了插入件 技术的进步，同时也间接推动了永磁体本身的发 展。典型的插入件发展史上三次革命都和永磁体研 究成果密切相关, 即: (1)1985年, 美国 Halbach 提出 新型永磁结构, 大大缩小了波荡器周期长度 ${ }^{[4]}$; (2) 1992 年, 日本 KEK 研制出高稳定永磁体真空室内 波荡器 ${ }^{[29]}$; (3)2004 年，日本 Spring-8 利用钕铁嗍低 温磁特性研制出 $\mathrm{CPMU}^{[30-31]}$, 大幅度提高了磁场 峰值。

我国对永磁体在同步辐射光源及自由电子激光 等尖端科学工程应用研究经验十分缺乏, 造成制备 的永磁体性能与质量具有 “偶然性” , 没有形成完 整的、系统性的高性能插入件永磁体设计与研制的 思想与方法。插入件用永磁体的应用基础研究具有 “共性特征”，将直接带动我国永磁体行业本身技术 的提升发展，同时对我国永磁体高端应用自主知识 产权化, 对中国制造永磁体走向国际大科学工程等 领域，对永磁体在国防、科研、医疗、信息、电力 和交通等各领域高水平应用有着重要推动作用。

\section{参考文献:}

[1] HIDEO ONUKI, PASCAL ELLEAUMe. Wigglers, Undulators and Their Applications. London: Taylor \& Francis, 2003, PART I: 87-132.

[2] 马礼敦, 杨福家. 同步辐射应用概论. 上海: 复旦大学出版社, 2001: 30-33.

[3] 麦振洪. 同步辐射光源及其应用. 北京: 科学出版社, 2013: 88-99.

[4] HALBACH K. Physical and optical Properties of rare earth cobalt magnets. Nuclear Instruments and Methods in Physics Research, 1981, 87: 09-117.

[5] 傅 萌. 基于 Halbach 阵列的高精度平面磁悬浮电机的设计和制 造. 南京: 东南大学硕士学位论文, 2007.

[6] 成玉卫. 基于 Halbach 结构的永磁电动悬浮技术研究. 长沙: 国 防科学技术大学硕士学位论文, 2009 .

[7] 刘 艳. 基于 HALBACH 阵列的盘式无铁心永磁同步电动机分析 与计算. 天津: 天津大学硕士学位论文, 2004.

[8] 钟文定. 技术磁学. 北京: 科学出版社, 2008: 1-10.

[9] 周寿增, 董清飞. 超强永磁体. 北京：治金工业出版社，1999: $1-15$.

[10] 尹 真, 编著. 电动力学. 北京: 科学出版社, 2012: 197-209.

[11] http://ssrf.sinap.ac.cn/

[12] ZHANG LING-YI, ZHUANG JIE-JIA, ZHAO KUI, et al. Fourth-generation light sources. High Power Laser and Particle Beams, 2001, 13(1): 51-55.

[13] 陈念. 相干谐波自由电子激光装置改进及模拟计算. 合肥：中国 科学技术大学博士学位论文, 2005 .

[14] 陆 杰. 上海光源首批插入件积分场测量及垫补. 上海：中科院 上海应用物理研究所博士学位论文, 2010.

[15] CHARLES KITEGI. Development of a Cryogenic Permanent Magnet Undulator at the ESRF. Doctoral Dissertafion, 2008.

[16] 张 伟. 新型螺旋型波荡器的物理研究. 上海: 中科院上海应用物 理研究所博士学位论文, 2011.

[17] 先进波荡器物理与技术. 第 142 期东方科技论坛, 2009. 12.03-04, 中国·上海.

[18] 超导与低温插入件技术. 第 197 期东方科技论坛, 2011.12.13-14, 中国·上海.

[19] 何永周. 低温永磁波荡器永磁体磁特性与样机磁场研究. 北京: 中国科学院大学博士学位论文, 2015.

[20] HE YONG-ZHOU, ZHANG JI-DONG, et al. Development of magnet blocks for SSRF initial undulators. High Power Laser And Particle Beams, 2010, 22(7): 1627-1630.

[21] HE YONG-ZHOU, ZHOU QIAO-GEN. The experiment research on $\mathrm{Sm}_{2} \mathrm{Co}_{17}$ magnets for SSRF In-vacuum undulators. High Power Laser and Particle Beams, 2012, 24(9): 2187-2192.

[22] HE YONG-ZHOU, ZHOU QIAO-GEN, ZHANG JI-DONG, et al. 
$\mathrm{Sm}_{2} \mathrm{Co}_{17}$ magnet blocks for the In-vacuum undulators (IVU20) at the SSRF. Chinese Physics C, 2011, 35(4): 392-396.

[23] HE YONG-ZHOU, WU HONG-PING, ZOU ZHI-QIANG. Magnetic properties of bulk polycrystalline $\operatorname{Pr}_{1-x} \mathrm{Nd}_{x}(\mathrm{x}=0,0.8) \mathrm{FeB}$ permanent magnets. Chinese Phys. B, 2014, 23(4): 047501.

[24] HE YONG-ZHOU. Experiment research on residual magnetization of rare earthe permanent magnet for cryogenic undulator. Chinese Phys. B, 2013, 22(7): 074101.

[25] HE YONG-ZHOU, ZHANG JI-DONG, ZHOU GIAO-GEN, et al. Measurement of the magnet blocks for SSRF insertion devices. AIP Conference Proceedings, 2010, 1234: 527-529.

[26] HE YONG-ZHOU. Inhomogeneity of external magnetic field for permanent magnet. Acta Phys. Sin., 2013, 62(8): 084105.

[27] CHEN NIAN, HE YONG-ZHOU, REN FENG-LIN, et al. Magnet measurement using 2D automatic rotation Helmholtz. High Power
Laser and Particle Beams, 2012, 24(8): 1941-1946.

[28] CHEN MING, LIU JUN-NAN, XUE SONG. Outgassing characteristics of graphite sheet in shanghai synchrotron radiation facility. Chinese Journal of Vacuum Science and Technology, 2013, 33(7): 661-663.

[29] SHIGERU YAMAMOTO, TATSURO SHIOYA, MASANORI HARA, et al. Construction of an in-vacuum type undulator for Production of undulator $\mathrm{X}$ rays in the $5-25 \mathrm{keV}$ region. Review of Scientific Instruments, 1992, 63: 400-405.

[30] CHAVANNE J, LEBEC G, PENEL C, et al. Cryogenic permanent magnet undulators. AIP Conference Proceedings, 2010, 1234: $25-28$.

[31] TANAKA T, SEIKE T, KAGAMIHATA A, et al. In-situ magnetic correction for cryogenic undulators. Physical Review Special Topics, 2009, 12(12): 1-8. 